\title{
The Effect of Helicity on Kinetic Energy Cascade in Compressible Helical Turbulence
}

\author{
Zheng Yan ${ }^{1,2}$, Xinliang $\mathrm{Li}^{1,2}$, Yaowei $\mathrm{Fu}^{1,2}$ and Changping $\mathrm{Yu}^{1,2 *}$ \\ ${ }^{1}$ LHD, Institute of Mechanics, Chinese Academy of Sciences, Beijing 100190, China \\ 2 School of Engineering Science, University of Chinese Academy of Sciences, \\ Beijing 100049, China
}

Received 11 August 2018; Accepted (in revised version) 6 December 2018

\begin{abstract}
Helicity plays an important role in the nonlinear dynamic process of compressible helical turbulence. We carry out direct numerical simulations (DNS) of compressible helical turbulence with different mean helicity at the grid resolution of $512^{3}$ and try to explore the physical mechanism of kinetic energy cascade under the effect of helicity. The filtering method of coarse-graining is employed for scale decomposition to get kinetic energy flux. We get some novel conclusions in contrast to pre-existing knowledge in incompressible helical turbulence. Firstly, helicity also hinders the process of compressible kinetic energy cascade and reduces viscous dissipation. Secondly, helicity weakens some exclusive features, such as coupling between compressible and solenoidal mode of fluctuating velocity, energy transformation between kinetic energy and internal energy, and shocklets which appear only in compressible turbulence and serves as the role of constraint.
\end{abstract}

AMS subject classifications: 76A02, 76F05, 76J20

Key words: Helicity, direct numerical simulations, kinetic energy cascade.

\section{Introduction}

Helicity has numerous interesting properties and attracts vast attention of theoretical, numerical and experimental investigations in the past few years. It's defined as

$$
H=\int_{V} \mathbf{u}(\mathbf{x}) \cdot \omega(\mathbf{x}) d^{3} \mathbf{x}
$$

where $\mathbf{u}(\mathbf{x})$ is the velocity and $\boldsymbol{\omega}(\mathbf{x})$ is the vorticity. Similar to kinetic energy of the Euler equation of ideal flows, this integral is also an invariant. It measures the degree of knottedness or linkage of the vortex lines [1]. Since helicity density defined as $h=\mathbf{u} \cdot \boldsymbol{\omega}$ is the

*Corresponding author.

Emails: yanzheng@imech.ac.cn (Z. Yan), lixl@imech.ac.cn (X. L. Li), fuyaowei@imech.ac.cn (Y. W. Fu), cpyu@imech.ac.cn (C. P. Yu) 
scalar product of a pure vector and a pseudovector, $h$ is a pseudoscalar. Its definition determines that it is a measure of chirality or mirror symmetry of the flow. So the difficulty of studying helicity always lies in choosing appropriate frame of reference [2].

In 1961, Betchov firstly introduced the concept of helicity to the context of turbulence [3]. He presented the general form of velocity correlations of two points in "semiisotropic" turbulence which means that statistical properties are invariant under rotation nor reflection. As following:

$$
\left\langle v_{i}(\mathbf{x}) v_{j}(\mathbf{x}+\mathbf{r})\right\rangle=\left\langle v^{2}\right\rangle\left(\frac{b_{r r}(\mathbf{r})-b_{t t}(\mathbf{r})}{r^{2}}\right) r_{i} r_{j}+b_{t t} \delta_{i j}+\epsilon_{i j k} g(\mathbf{r}) r_{k}
$$

where $b_{r r}$ and $b_{t t}$ are longitudinal and transversal correlation function defined in classical isotropic turbulence theory. Last term in Eqs. (1.2) is not invariant under reflection and it is related to helicity density by $\langle h(\mathbf{x})\rangle=-6 g(0)$. Hence helicity is related to the symmetry properties of turbulence, and it might change some properties of turbulence.

In the past few decades, the studies of helicity involve the influence on non linear term in Naiver-Stokes equations (NSEs) [4-8], the mechanism of energy dissipation [9, 10], intermittency and energy cascade [11-16,20-22] in incompressible turbulence.

Some researches of helicity originate from non linear term in NSEs. Kraichnan et al. simulated decaying isotropic turbulence initialized from Gaussian distributed velocity field [4]. They found that the depression represented by normalized mean-square non linear term is up to $57 \%$ contrast to initial status, and predicted that the depression is dynamically generic. Shtilman et al. investigated the reduction mechanism of viscous dissipation raised by Kraichnan [5]. They found two sources accounting for this phenomenon. One is the alignment of velocity and vorticity, and the other is a tendency of Lamb vector to align with wave vector in Fourier space. Tsinober et al. compared nonlinearity in regions dominated by enstrophy and strain respectively [6]. It revealed that nonlinearity is strongly depressed by enstropy rather than strain. Bos et al. obtained a closed expression of the normalized mean-square nonlinearity through the direct interaction approximation [7]. They found that its scaling in the inertial subrange is dominated by the sweeping of the energetic scales rather than random sweeping scales, and the depletion of nonlinearity is a constant in the inertial range. They also simulated decaying two-dimensional turbulence [8], and confirmed that the depression of nonlinearity could be divided into two steps. The first is a fast relaxation whose timescale is the order of the time of vortical structures, the second is a slow relaxation whose timescale is that large-scale eddies breakdown to viscous-scale eddies, and both steps are independent of Reynolds number.

The joint cascade of kinetic energy and helicity has elicited increasing interest because helicity affects the process of kinetic energy cascade and represents flow structure by itself. Lesieur et al. carried out numerical simulations of three-dimension homogeneous and isotropic turbulence at very high Reynolds number by using the method of EddyDamped Quasi-Normal (EDQN) theory [9], and they revealed that there exists a joint cascade of energy and helicity. It is shown that the helicity cascade hinders energy cas- 